\title{
A Survey on Intra and Inter Domain Reusability Routing Protocols in MANETs
}

\author{
Ravikiran K. \\ ANUCET, Guntur, A.P.
}

\author{
Sudhakar Nagalla \\ Professor, \\ Bapatla Engineering College, \\ Bapatla, Guntur, Andrapradesh.
}

\begin{abstract}
In any network both wired or wireless network transmutation of data from source to destination is vital and it is also important that has to be transmuted in less time. In wired network it is not a problem because it is connection oriented and high bandwidth is available. But in wireless network it is crucial to select a path which is lossless and minimize the time because it is connection less network there is more chance to loss a link. In recent days there are number of routing protocols were developed but they are not guaranty high through put. Here we studied number of such routing protocols and we give a conclusion that if we consider spatial or domain usability as consideration in implementation of routing protocols then we will get maximum through put. Spatial or domain reusability-aware single-path routing (SASR) and any path routing (SAAR) protocols are the two routing protocols which will give maximum through put in single and any path respectively.
\end{abstract}

\section{General Terms}

Networks, Routing, Algorithms.

\section{Keywords}

Wireless network, protocol, Inter Domain, intra Domain, MANT

\section{INTRODUCTION}

Remote systems are a rising innovation that will enable clients to get to data and administrations electronically from anyplace. A specially appointed remote system contains number of hubs which are having ability of portability. The system topology will change powerfully. For move of information from source hub to goal hub we can't utilize customary directing conventions which are utilized as a part of wired organize. So for that an assortment of steering conventions is created for specially appointed remote system.

As of late, an expansive number of directing conventions have been proposed for remote correspondence [1,2] Nonetheless, crucial issue with existing remote steering conventions is that limiting the general number (or time) of transmissions to convey a solitary parcel from a source hub to a goal hub

\section{LITERATURE SURVEY}

For this survey first we consider the classification of ad-hoc routing protocols. Routing Protocols in mobile ad-hoc wireless network can be classified into different categories [2] based upon some criteria. So for the classification of routing protocols in wireless network we need a new methodology. In generally the classification was done by dividing protocols based on Use of temporal information for routing, based upon Routing information update mechanism, and Utilization of some specific resources. In MANETs the routing protocols can be classified into 3 Categories depends upon the update mechanism of routing information. They are: table-driven routing protocols or Proactive, on-demand routing protocols or Reactive and Hybrid routing protocols, Based on the use of temporal information used for routing the routing protocols can be classified as Routing protocols using past temporal information and routing protocols that use future temporal information. In internet we use hierarchical routing topology in order to reduce the Routing topology being used because it will decrease the information maintained at base routers. Flat topology routing protocol and crass based or Hierarchical topology routing protocols, Depends upon Utilization of Unique Resources the protocols can be categorized into Power-aware routing and Geographical information assisted routing. The below figure shows The classification of above sed protocols.

These are the following papers studied for this survey on intra and inter domain reusability routing protocols in mobile ad hoc networks

\subsection{Dynamic source routing in ad hoc wireless networks by $D$. B. Johnson and D. A. Maltz}

In Dynamic Source Routing [3] there are two mechanisms: Route Discovery and Route Maintenance Route Discovery mechanism is similar to the one in AODV for Route Maintenance Two types of packets used: Route Error Packet and Acknowledgement good for maintaining network connectivity under frequent changes in topology High overhead is unnecessary in networks with static nodes. Even though the performance of this routing protocol is good in low-mobility of nodes and static topology environments, if the moving of the nodes are high then the performance will be degrades rapidly. And it also considers routing overhead due to the source-routing mechanism employed in DSR. In the dynamic source routing protocol the routing overhead is directly proportional to the length of the path. The figure 2 shows the route establishment in DSRP. 


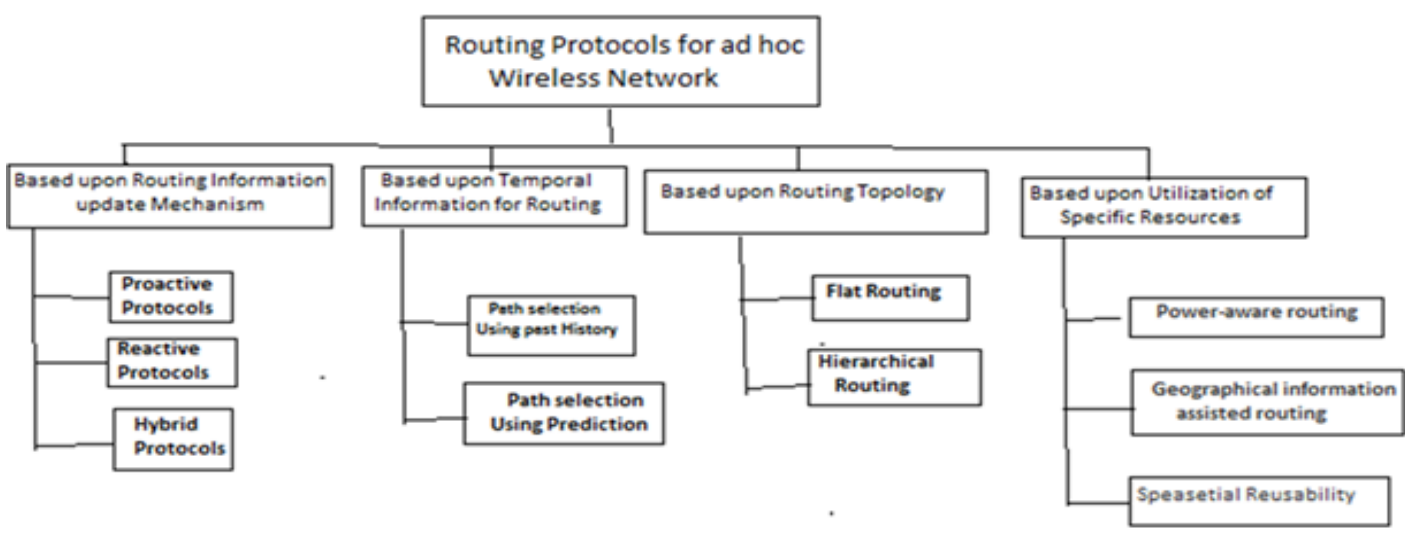

Fig 1: Classification of Routing Protocols in Wireless ad hoc network

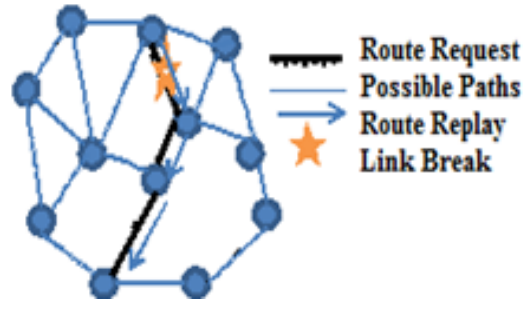

Fig 2: Route Establishments in DSRP

\subsection{Ad Hoc On Demand Distance Vector Routing by C.E. Perkins and E.M. Royer}

Ad hoc on-demand distance vector (AODV) [4] routing protocol uses an on-demand approach for finding routes to find a path to the destination, the source broadcasts a route request packet. The adjacent nodes broadcast the packet until it reaches an intermediate node that has recently route information about the destination or till it reaches the destination but here there is problem to get loops to avoid it will use sequence number. The sequence number is used to avoid loops in the network topology and make sure that wither the intermediate node is replay to the route request and replay must be with the newly updated information only. When the source node moves then it can reinitiate route discovery to the destination node. If any one of the intermediate nodes move away from the region then the moved nodes adjacent nodes realizes the path failure and sends the path failure information to its upstream neighbors and it continue the process up to the source can reinitiate route discovery if necessary

The following figures 3 and 4 shows the route Establishment and Route Maintais in the ad hoc on demand routing protocols.

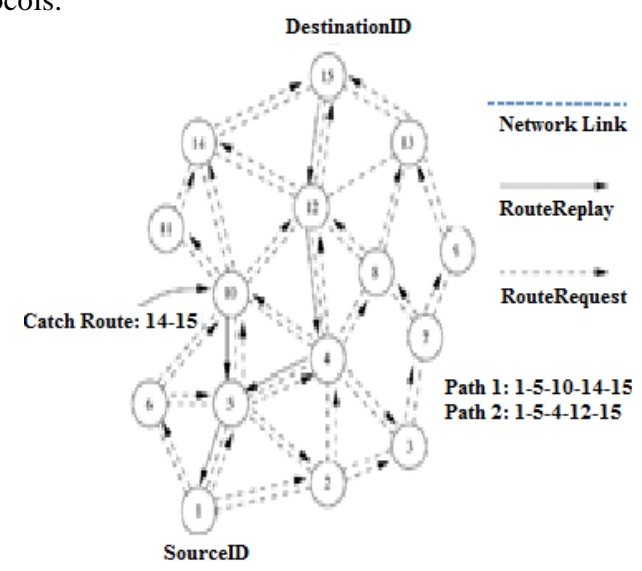

Fig 3: Route Establishments in AODVP.

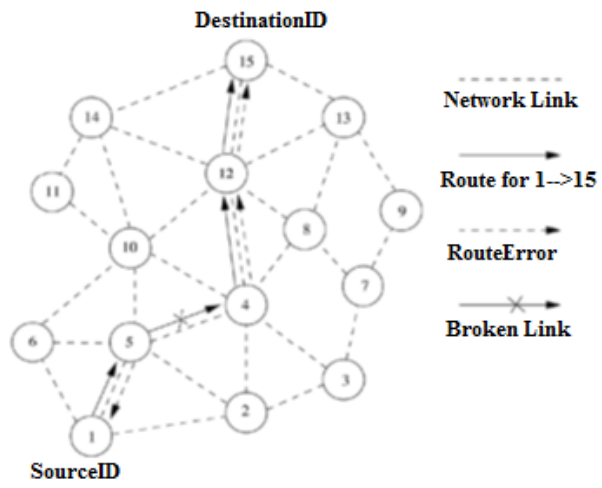

Fig 4: Route Maintenance in AODVP

\subsection{Temporally Ordered Routing \\ Algorithm by Park and Corson}

Temporally ordered routing algorithm (TORA) [5] is a source-initiated on demand routing protocol which uses a link reversal algorithm and provides loop-free multipath routes to a destination node. This routing protocol is mainly proposed for highly dynamic mobile multi hop a-hoc wireless networks. The TORA routing protocol finds number of paths from source hope to destination hope. One of The important feature of TORA is that the control messages are very near the occurrence of a topological change. To achieve the above goal every nodes maintain routing information about all adjacent nodes. There are three basic functions are there for TORA routing protocol for route discovery. The functions are Route creation, Route maintenance, and Route termination. When the detection of partition and subsequent deletion of paths taken at same time then it may cause temporary oscillations and transient loos. The local reconfiguration of routs results in non-optimal paths.

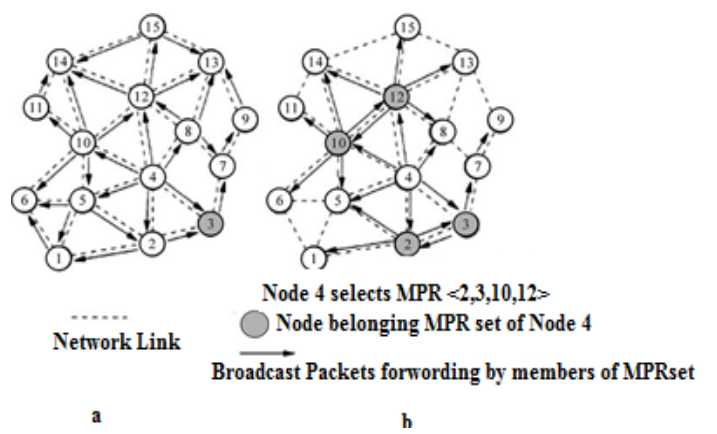

Fig 5: Routing Establishment in TORA a)Flooding the Network takes as many as transitions as the number of nodes b) Flooding the Entire network with six transmission using MPR scheme 


\subsection{J. Broch, and D. A. Maltz "A performance comparison of multi-hop wireless ad hoc network routing protocols"}

In this paper [6] creators think about execution of various multi-bounce directing conventions, for example, DSDV, TORA, DSR and AODV, and They gives those conventions brings about reenactment condition. When looking at the quantity of steering overhead parcels sent by each of the conventions, DSR obviously squander least overhead. AODVLL utilizes a Course Disclosure system in view of DSR's, however it makes bounce by-jump steering state in every hub along a way keeping in mind the end goal to wipe out the overhead of source directing from information parcels. They have stretched out system test system to precisely demonstrate the Macintosh and physical-layer conduct of the IEEE 802.11 remote LAN standard, including a sensible remote transmission channel model, and present the aftereffects of reenactments of systems of 50 versatile hubs.

\subsection{Exor: Opportunistic multi-hop routing for wireless network by $S$ Biswas and $R$ Morris}

ExOR [7] is a coordinated directing and Macintosh method that deftly misuses the communicate attributes of the remote condition. Not at all like the customary steering conventions, where the best arrangement of hubs from a source to a goal is chosen before parcels are directed along that way, ExOR concedes the following bounce choice until it knows every one of the hubs that got a bundle and enables the nearest hub to the goal to be the following jump and retransmit that bundle toward goal. Therefore, ExOR can attempt numerous long however lossy connections simultaneously, bringing about high expected advance per transmission.

\subsection{A multi radio unification protocol for}

IEEE 802.11 wireless networks by A.

Adya, P. Bahl, J. Padhye, A. Wolman, and L. Zhou.

This paper [8] presents a link layer protocol called the Multiradio Unification Protocol or MUP. On a one hub or node, Multiradio Unification Protocol correlative the operation of varies wireless network cards tuned to without overlapping frequency channels that is without interference. The main objective of MUP is to upgrade local spectrum usage by choosing an adequate tunnel or channel in a multi hop wireless network. It low the overhead of the implementation and design of MUP. Here the authors analyze its performance by implementing in network simulation. These Results show the MUP significantly improves both TCP throughput and user perceived latency for realistic workloads in dynamic traffic patterns with realistic topologies. They plan to investigate other metrics for channel quality, a more scalable method for sending probes using broadcasts, and the impact of mobile nodes on MUP.

\subsection{Routing in multi-radio, multi hop wireless mesh networks by $\mathbf{R}$. Draves, J. Padhye, and B. Zill}

This paper [9] presents a connection layer convention called the Multi-radio Unification Convention or MUP. On a solitary hub, MUP arranges the operation of different remote system cards tuned to non-covering recurrence channels. The fundamental point of MUP is to upgrade neighborhood range use by picking a proficient direct in a multi jump remote system. It low the overhead of the usage and plan of MUP. Here the creators examine its execution by actualizing in system reenactment. These Outcomes demonstrate the MUP essentially enhances both TCP throughput and client saw inertness for practical workloads in powerful activity designs with reasonable topologies. They plan to examine different measurements for channel quality, a more adaptable technique for sending tests utilizing communicates, and the effect of portable hubs on MUP.

\subsection{N. M. Jones, B. Shrader, and E. Modiano, Optimal routing and scheduling for a simple network coding scheme}

Arrange coding [10], initially presented in, can build organize throughput by enabling middle of the road hubs to join or encode the information they get, as opposed to just imitating and sending it. This paper considers together ideal steering, booking, and system coding methodologies to expand throughput in remote systems. It presents k-tuple coding, a speculation of pairwise coding with next-jump decidability, and completely describe the area of entry rates for which the system lines can be balanced out under this coding technique. Creators assessed the LCMFrame strategy by means of bundle reproduction and LP assessment for pairwise and 3-tuple coding.

\subsection{Spatial Reusability-Aware Routing in Multi- Hop Wireless Networks by Tong Meng, Fan Wu, Zheng Yang and \\ Athanasios V. Vasilakos.}

This paper [11] presents spatial or domain reusability of the remote correspondence media to enhance the end-to-end throughput for that they are having two conventions Spatial or domain reusability-aware single-path routing (SASR) and any path routing (SAAR) protocols. Assessment comes about demonstrate that protocols essentially enhance the end-to-end throughput which they developed and existed protocols. This paper tells one bearing to additionally investigate chances to enhance the execution of directing calculations by breaking down uncommon failing to meet expectations cases distinguished in the assessment and streamline framework wide execution.

\section{PIT FALLS IN EXISTING ROUTING PROTOCOLS}

In MANETS the following issues are identified as some major pit falls in the existing routing protocols by analyzing the above mentioned paper.

1. Power consumption is the main challenge in wireless network.

2. In the existing routing protocols they consider shortest path but ignore the throughput.

3. These protocols are not considering secure data transmission with less cost.

4. One of the main problems with Existing routing protocols is minimizing the number of hops or time but lave maximum end-to-end throughput.

5. Up to my knowledge these routing protocols are no consider special or domain usability into account. 
6. All the existing routing protocols are not considering interference.

7. There is a need of centralized control for MAC layer scheduling and to avoid transmission contention.

\section{DOMAIN REUSABILITY}

Domain Reusability implies at least two connections having a similar recurrence direct in the same spatial region in the meantime. In late day the scientists are more focus on spatial or space Reusability and they demonstrated that on the off chance that we consider spatial or area reusability into record in the usage of steering conventions we will get most extreme through put. To demonstrate this, the creators considered one of the principle metric Expected transmission count (ETX). The howl illustration demonstrates the creator's usage case.

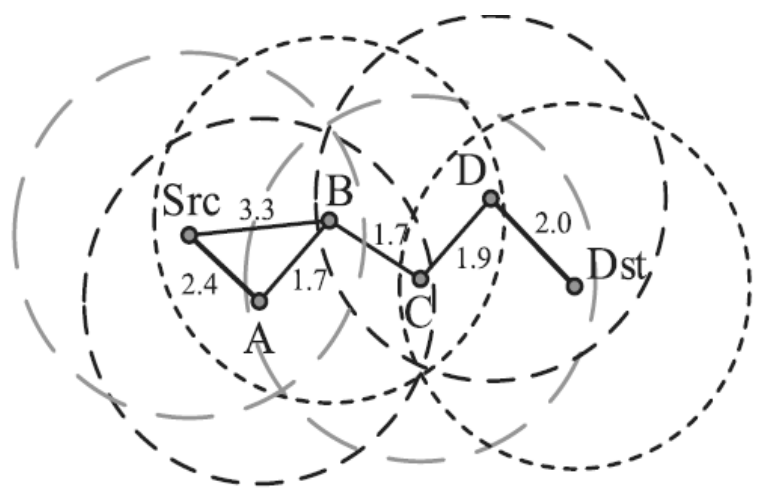

Fig 6: An Example of Network

As appeared in the above figure there are two ways from hub Src (source) to hub Dst (Destination)

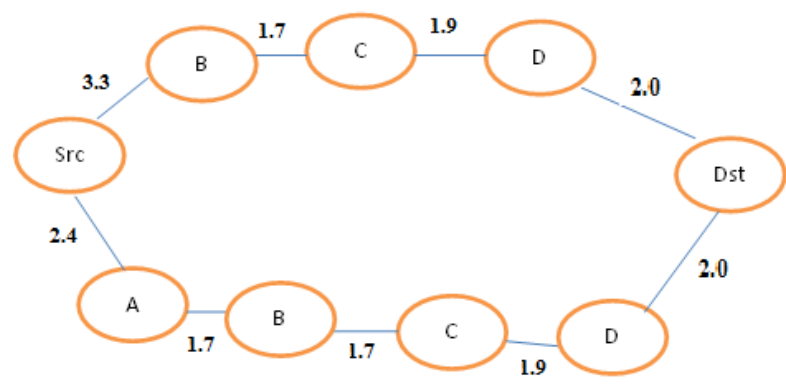

Fig 7: Two Possible Paths for the network

Way I: Src -B-C-D- Dst $(3.3+1.7+1.9+2.0=8.9)$

Way II: Src -A-B-C-D- Dst $(2.4+1.7+1.7+1.9+2.0=9.7)$

The ETX cost of way I and way II 8.9 and 9.7, individually. Since way I has a littler ETX taken a toll, it is regularly chosen by customary ETX-based steering conventions, and is normal to have better execution. In any case, the authors reproduction comes about demonstrate that way II accomplishes a normal end-to-end throughput of $753 \mathrm{Kbps}$, which is 10.2 percent higher than $683 \mathrm{Kbps}$ accomplished by way I, when the transmission rate is $11 \mathrm{Mbps}$.

\section{CONCLUSION}

In any network both wired or wireless network transmutation of data from source to destination is vital and it is also important that has to be transmuted in less time. By studding the above papers we given the conclusion that if we consider spatial or domain usability as consideration in implementation of routing protocols then we will get maximum through put. Spatial or domain reusability-aware single-path routing (SASR) and any path routing (SAAR) protocols are the two routing protocols which will give maximum through put in single and any path respectively.

\section{FUTURE SCOPE}

As for the future work, one direction is to further explore opportunities to improve the performance of routing algorithms by analyzing special or Domain reusability by considering it in inter domain reusability and another way to extend this work by considering some new routing metrics such s number of inter networks between source network to destination network.

\section{REFERENCES}

[1] Yan Jhang,J Luo,and Honglin "Wireless Mesh Networking" Auer batch Publications .

[2] "Ad hoc wireless networks Architectures and Protocols" a text book by C. Siva Ram Murthy and B. Manoj.

[3] D. B. Johnson and D. A. Maltz, "Dynamic source routing in ad hoc wireless networks". 1996

[4] C. E. Perkins and E. M. Belding-Royer, "Ad-hoc ondemand distance vector routing," in IEEE Proceedings. Appl., 1999.

[5] Park and Corson "Temporary-ordered Routing Algorithm (TORA)" 2001 Internet Draft.

[6] J. Broch, and D. A. Maltz "A performance comparison of multi-hop wireless ad hoc network routing protocols".

[7] S. Biswas and R. Morris, "Exor: Opportunistic multi-hop routing for wireless networks,"

[8] A. Adya, P. Bahl, J. Padhye, A. Wolman, and L. Zhou, "A multi radio unification protocol for IEEE 802.11 wireless networks".

[9] R. Draves, J. Padhye, and B. Zill, "Routing in multiradio, multihop wireless mesh networks"

[10] N. M. Jones, B. Shrader, and E. Modiano, "Optimal routing and scheduling for a simple network coding scheme".

[11] Tong Meng, Fan Wu, ZhengYang,and Athanasios V. Vasilakos,"Spatial Reusability-Aware Routing in MultiHop Wireless Networks". 